\title{
SOME OPEN MAPPING THEOREMS FOR MEASURES
}

\author{
BY
}

\author{
SEYMOUR Z. DITOR AND LARRY Q. EIFLER
}

\begin{abstract}
Given a compact Hausdorff space $X$, let $C(X)$ be the Banach space of continuous real valued functions on $X$ with sup norm and let $M(X)$ be its dual considered as finite regular Borel measures on $X$. Let $U(X)$ denote the closed unit ball of $M(X)$ and let $P(X)$ denote the nonnegative measures in $M(X)$ of norm 1 . A continuous map $\varphi$ of $X$ onto another compact Hausdorff space $Y$ induces a natural linear transformation $\pi$ of $M(X)$ onto $M(Y)$ defined by setting $\pi(\mu)(g)=\mu(g \circ \varphi)$ for $\mu \in M(X)$ and $g \in C(Y)$. It is shown that $\pi$ is norm open on $U(X)$ and on $A \cdot P(X)$ for any subset $A$ of the real numbers. If $\varphi$ is open, then $\pi$ is weak* open on $A \cdot P(X)$. Several examples are given which show that generalization in certain directions is not possible. The paper concludes with some remarks about continuous selections.
\end{abstract}

1. Introduction. Let $E_{1}$ and $E_{2}$ be Banach spaces with closed unit balls $U_{1}$ and $U_{2}$, respectively, and let $\pi$ be a linear transformation of $E_{1}$ onto $E_{2}$ which carries $U_{1}$ onto $U_{2}$. Of course, $\pi$ is an open map of $E_{1}$ onto $E_{2}$, but there are simple examples which illustrate that $\pi$ is not necessarily open as a map of $U_{1}$ onto $U_{2}$ (see $\S$ ). It can be shown, though, that if $U_{1}$ is sufficiently round (precisely, locally uniformly convex $\left[1\right.$, p. 113]), then $\pi$ will be open on $U_{1}$. However, as indicated by the examples in $\S 5$, in the absence of any roundness conditions, general theorems of this kind are hard to come by.

We shall be concerned with open mapping theorems in the following setting. Let $X$ be a compact Hausdorff space and let $C(X)$ be the Banach space of continuous real-valued functions on $X$ with the sup norm. The dual of $C(X)$ is the space $M(X)$ of real regular Borel measures on $X$ of finite total variation. Denote the closed unit ball in $M(X)$ by $U(X)$, the nonnegative (resp. nonpositive) measures in $M(X)$ by $M^{+}(X)$ (resp. $M^{-}(X)$ ), and the measures in $M^{+}(X)$ of norm 1 by $P(X)$ (the probability measures on $X$ ). Given another compact Hausdorff space $Y$ and continuous map $\varphi$ of $X$ onto $Y$, there is a natural linear transformation $\pi$ of $M(X)$ onto $M(Y)$ which takes $\mu \in M(X)$ onto the measure $\pi(\mu)$ defined on the Borel sets $B$ of $Y$ by

$$
\pi(\mu)(B)=\mu\left(\varphi^{-1}(B)\right)
$$

or, equivalently, on the functions $g \in C(Y)$ by

$$
\pi(\mu)(g)=\mu(g \circ \varphi) .
$$

Received by the editors June 23, 1970.

AMS 1969 subject classifications. Primary 2846, 4625; Secondary 4606, 4610.

Key words and phrases. Open mappings, measures on compact spaces, probability measures, continuous selections. 
The map $\pi$ is the dual of tice isometric embedding of $C(Y)$ into $C(X)$ which takes $g \in C(Y)$ into $g \circ \varphi$. It follows from the Hahn-Banach theorem, then, that $\pi(U(X))$ $=U(Y)$. Moreover, since $\pi$ is a positive operator and $\pi(\mu)(1)=\mu(1)$, we have $\pi(P(X))=P(Y)$, and, consequently, $\pi\left(M^{+}(X)\right)=M^{+}(Y)$. In fact, $\pi(A \cdot P(X))$ $=A \cdot P(Y)$ for any set $A$ of real numbers.

It is shown in $\$ 2$ that, in either the norm or weak* topologies, $\pi$ is open on $M^{+}(X)$ if and only if $\pi$ is open on $A \cdot P(X)$ for every subset $A$ of $R$, the real numbers. We then show in $\S 3$ that $\pi$ is norm open on $U(X)$ and on $M^{+}(X)$. In $\S 4$, we show that $\pi$ is weak* open on $M^{+}(X)$ (or $P(X)$ ) if and only if $\varphi$ is open. One of the examples shows that $\pi$ is generally not weak* open on $U(X)$, even if $\varphi$ is open. We conclude with some remarks about selection theorems.

The authors wish to thank A. J. Lazar for several useful conversations concerning this research.

2. Some preliminary remarks. In either the norm or weak* topologies, it is clear that a subset $V$ of $M^{+} \cup M^{-}$is open in $M^{+} \cup M^{-}$if and only if $V \cap M^{+}$ is open in $M^{+}$and $V \cap M^{-}$is open in $M^{-}$. Hence, $\pi$ is open on $M^{+}(X)$ (or $\left.M^{-}(X)\right)$ if and only if $\pi$ is open on $M^{+}(X) \cup M^{-}(X)$. Moreover, for any subset $V$ of $M^{+}(X) \cup M^{-}(X)$ and any $A \subset R$,

$$
\pi(V \cap A \cdot P(X))=\pi(V) \cap A \cdot P(Y) .
$$

Hence, we have the following.

LemMa 2.1. In either the norm or weak* topologies, the map $\pi$ is open on $\mathrm{M}^{+}(X)$ if and only if $\pi$ is open on $A \cdot P(X)$ for every set $A$ of real numbers.

For any nonnegative measure $\mu_{0}$, let $\left[0, \mu_{0}\right]$ denote the set of all measures $\mu$ satisfying $0 \leqq \mu \leqq \mu_{0}$.

LEMmA 2.2. For any $\mu_{0} \in M^{+}(X), \pi\left[0, \mu_{0}\right]=\left[0, \pi\left(\mu_{0}\right)\right]$.

Proof. Since $\pi$ is positive, $\pi\left[0, \mu_{0}\right] \subset\left[0, \pi\left(\mu_{0}\right)\right]$. Moreover, $\left[0, \mu_{0}\right]$ is a weak* compact convex subset of $M(X)$ and $\pi$ is weak* continuous. Hence, $\pi\left[0, \mu_{0}\right]$ is a weak* compact convex subset of $M(Y)$. Therefore, if $0 \leqq \nu \leqq \pi\left(\mu_{0}\right)$ and it is assumed that $\nu \notin \pi\left[0, \mu_{0}\right]$, then, by the separation theorem, there exists $g \in C(Y)$ and $\varepsilon>0$ such that, for all $\mu \in\left[0, \mu_{0}\right]$,

$$
\int g d \nu \geqq \varepsilon+\int g \circ \varphi d \mu .
$$

Let $S=\{x \in X: g(\varphi(x)) \geqq 0\}$ and let $\omega=\mu_{0} \mid S$. Then $\omega \in\left[0, \mu_{0}\right]$ and

$$
\int g \circ \varphi d \omega=\int g^{+} d \pi\left(\mu_{0}\right) \geqq \int g d \nu,
$$

which is a contradiction. 
REMARK. Lemma 2.2 is, in fact, a special case of the following result. Let $F$ be a Banach space and vector lattice and let $E$ be a closed vector sublattice. If $\left\{f^{+}: f \in F,\|f\|=1\right\}$ is norm bounded, then the restriction map $\pi$ of $F^{*}$ onto $E^{*}$ maps intervals onto intervals. (Given $\mu_{0} \geqq 0$ in $F^{*}$ and $\nu \in\left[0, \pi\left(\mu_{0}\right)\right]$ in $E^{*}$, define the sublinear functional $p$ on $F$ by $p(f)=\mu_{0}\left(f^{+}\right)$and apply the Hahn-Banach theorem.)

3. Open mapping theorems for the norm topology. For any subset $K$ of $M(X)$, $\pi$ is open on $K$ if and only if $\pi$ is open at every point $\mu$ in $K$, i.e., for every neighborhood $V$ of $\mu$ in $K, \pi(V)$ is a neighborhood of $\pi(\mu)$ in $\pi(K)$. For the norm topology, this is equivalent to saying that for every sequence $\nu_{n}$ in $\pi(K)$ which converges in norm to $\pi(\mu)$, there is a sequence $\mu_{n}$ in $K$ which converges in norm to $\mu$ and $\pi\left(\mu_{n}\right)=\nu_{n}$ for all $n$.

THEOREM 3.1. $\pi$ is a norm open mapping of $M^{+}(X)$ onto $M^{+}(Y)$.

Proof. Fix $\mu_{0} \in M^{+}(X)$ and let $\nu_{0}=\pi\left(\mu_{0}\right)$. Suppose $\nu_{n} \in M^{+}(Y)$ and $\nu_{n} \rightarrow v_{0}$. Since $\pi$ is open on $M(X)$, there exist $\omega_{n} \rightarrow \mu_{0}$ and $\pi\left(\omega_{n}\right)=\nu_{n}$. Then $\pi\left(\omega_{n}^{+}\right) \geqq \nu_{n}$ and, by norm continuity of the lattice operations, $\omega_{n}^{+} \rightarrow \mu_{0}$. By Lemma 2.2 , there exist $\mu_{n} \in\left[0, \omega_{n}^{+}\right]$satisfying $\pi\left(\mu_{n}\right)=\nu_{n}$. Since $\omega_{n}^{+}-\mu_{n} \geqq 0$ and $\pi$ is an isometry on $M^{+}(X)$, we have

$$
\left\|\omega_{n}^{+}-\mu_{n}\right\|=\left\|\omega_{n}^{+}\right\|-\left\|\mu_{n}\right\|=\left\|\omega_{n}^{+}\right\|-\left\|\nu_{n}\right\|,
$$

which converges to $\left\|\mu_{0}\right\|-\left\|\nu_{0}\right\|=0$. It follows that $\mu_{n} \rightarrow \mu_{0}$. Hence, on $M^{+}(X), \pi$ is open at $\mu_{0}$.

THEOREM 3.2. $\pi$ is a norm open mapping of $U(X)$ onto $U(Y)$.

Proof. Fix $\mu_{0} \in U(X)$ and let $\nu_{0}=\pi\left(\mu_{0}\right)$.

Suppose $\left\|\nu_{0}\right\|<1$ and let $V$ be any open neighborhood of $\mu_{0}$ in $U(X)$. Then for some $\omega_{0}$ in $U(X), \pi\left(\omega_{0}\right)=\nu_{0}$ and $\left\|\omega_{0}\right\|=\left\|\nu_{0}\right\|$, and for some real number $t$ in the open interval $(0,1), \rho=t \omega_{0}+(1-t) \mu_{0}$ is in $V$. Since $\pi$ is open on $M(X), \pi(\rho)=\nu_{0}$, and $\|\rho\|<1$, it follows that $\pi(V)$ is a neighborhood of $\nu_{0}$.

Now assume $\left\|\nu_{0}\right\|=1$. Then $\left\|\mu_{0}\right\|=1$. Suppose $\nu_{n} \in U(Y)$ and $\nu_{n} \rightarrow \nu_{0}$. Since $\pi$ is open on $M(X)$, there exists $\omega_{n}$ in $M(X)$ such that $\omega_{n} \rightarrow \mu_{0}$ and $\pi\left(\omega_{n}\right)=\nu_{n}$. We modify the $\omega_{n}$ 's to bring them into $U(X)$. Since $\nu_{n}=\pi\left(\omega_{n}^{+}\right)-\pi\left(\omega_{n}^{-}\right)$, it follows that $\pi\left(\omega_{n}^{+}\right) \geqq \nu_{n}^{+}$and $\pi\left(\omega_{n}^{-}\right) \geqq \nu_{n}^{-}$. By Lemma 2.2 , there exists $\rho_{n} \in\left[0, \omega_{n}^{+}\right]$and $\lambda_{n} \in\left[0, \omega_{n}^{-}\right]$ satisfying $\pi\left(\rho_{n}\right)=\nu_{n}^{+}$and $\pi\left(\lambda_{n}\right)=\nu_{n}^{-}$. Set $\mu_{n}=\rho_{n}-\lambda_{n}$. Then $\pi\left(\mu_{n}\right)=\nu_{n}$ and $\mu_{n} \in U(X)$ since

$$
\left\|\mu_{n}\right\| \leqq\left\|\rho_{n}\right\|+\left\|\lambda_{n}\right\|=\left\|\nu_{n}^{+}\right\|+\left\|\nu_{n}^{-}\right\|=\left\|\nu_{n}\right\| \leqq 1 .
$$

Moreover, since $0 \leqq \rho_{n} \leqq \omega_{n}^{+}$and $0 \leqq \lambda_{n} \leqq \omega_{n}^{-}$,

$$
\left\|\omega_{n}-\mu_{n}\right\| \leqq\left\|\omega_{n}^{+}-\rho_{n}\right\|+\left\|\omega_{n}^{-}-\lambda_{n}\right\| \leqq\left\|\omega_{n}^{+}\right\|-\left\|\rho_{n}\right\|+\left\|\omega_{n}^{-}\right\|-\left\|\lambda_{n}\right\|=\left\|\omega_{n}\right\|-\left\|\nu_{n}\right\| .
$$

Hence, $\mu_{n} \rightarrow \mu_{0}$ and, on $U(X), \pi$ is open at $\mu_{0}$. 
4. Open mapping theorems for the weak* topology. In this section, we prove the equivalence of the three statements: (i) $\pi$ is weak* open on $P(X)$, (ii) $\varphi$ is open, and (iii) $\pi$ is weak* open on $M^{+}(X)$. By Lemma 2.1 , it suffices to prove that (i) $\Rightarrow$ (ii) $\Rightarrow$ (iii).

Proposition 4.1. If $\pi$ is a weak* open map of $P(X)$ onto $P(Y)$, then $\varphi$ is open.

Proof. Consider any $x_{0} \in X$ and let $y_{0}=\varphi\left(x_{0}\right)$. Let $f \in C(X)$ satisfy $f\left(x_{0}\right)=0$ and $f \geqq 0$. Set

$$
G=\{x \in X: f(x)<1\} \text { and } V=\{\mu \in P(X): \mu(f)<1\} .
$$

Since the sets $G$ obtained in this manner form a neighborhood base at $x_{0}$, it suffices to show that $\varphi(G)$ is a neighborhood of $y_{0}$. But $\pi(V)$ is a weak* neighborhood of $\delta_{y_{0}}$, the unit point mass at $y_{0}$. Since for any $\nu \in P(Y)$ and $g \in C(Y)$,

$$
\left|\nu(g)-g\left(y_{0}\right)\right| \leqq \nu\left(\left|g-g\left(y_{0}\right)\right|\right),
$$

it follows that there exist finitely many functions $g_{1}, \ldots, g_{n}$ in $C(Y)$ such that $g_{i}\left(y_{0}\right)=0, g_{i} \geqq 0$, for $i=1, \ldots, n$, and $W \subset \pi(V)$, where

Set

$$
W=\left\{\nu \in P(Y): \nu\left(g_{i}\right)<1, i=1, \ldots, n\right\} .
$$

$$
H=\left\{y \in Y: g_{i}(y)<1, i=1, \ldots, n\right\} .
$$

Then $H$ is a neighborhood of $y_{0}$ and $W \subset \pi(V)$ implies that $H \subset \varphi(G)$. For, if $y \in H$, then $\delta_{y} \in W$, so that $\delta_{y}=\pi(\mu)$ for some $\mu \in V$. Hence, $\mu$ is supported on $\varphi^{-1}(y)$ and $\mu(f)<1$, which implies that $f(x)<1$ for some $x \in \varphi^{-1}(y)$.

In the proofs given below, we require the notion of set convergence. The definitions and basic facts required are as follows (see [2]). Let $K$ be a topological space and let $F(K)$ denote the closed nonempty subsets of $K$. Given finitely many open sets $V_{1}, \ldots, V_{n}$ in $K$, let $\left\langle V_{1}, \ldots, V_{n}\right\rangle$ denote the collection of sets $A$ in $F(K)$ satisfying $A \subset \bigcup_{i=1}^{n} V_{i}$ and $A \cap V_{i} \neq \varnothing$ for $i=1, \ldots, n$. The sets of the form $\left\langle V_{1}, \ldots, V_{n}\right\rangle$ constitute a base for a topology on $F(K)$, called the finite topology. If $K$ is compact Hausdorff, then $F(K)$ is compact Hausdorff, and if $A_{\alpha} \rightarrow A$ in $F(K)$, for some net $A_{\alpha}$, then $A$ is the set consisting of those points $a \in K$ for which $V \cap A_{\alpha} \neq \varnothing$ eventually, for every neighborhood $V$ of $a$.

LemMa 4.2. If $K$ is a compact subset of a linear topological space and $A_{\alpha}$ is a net of closed convex subsets of $K$ which converge to a set $A$ in the finite topology, then $A$ is convex.

Proof. If $\mu, \nu \in A$ and $0 \leqq t \leqq 1$, then any neighborhood $W$ of $t \mu+(1-t) \nu$ contains $t U+(1-t) V$ for some neighborhoods $U, V$ of $\mu, \nu$ respectively. Since $U \cap A_{\alpha}$ and $V \cap A_{\alpha}$ are eventually nonempty and each $A_{\alpha}$ is convex, it follows that $W \cap A_{\alpha}$ is eventually nonempty. Hence, $t \mu+(1-t) \nu \in A$. 
Lemma 4.3. Suppose $\varphi$ is an open map of $X$ onto $Y$. Then for each $f \in C(X)$, the real-valued function $f^{*}$ defined on $Y$ by

is continuous.

$$
f^{*}(y)=\sup \{f(x): \varphi(x)=y\}
$$

Proof. Let $r$ be any real number. Then

$$
\left\{y \in Y: f^{*}(y)>r\right\}=\varphi(\{x \in X: f(x)>r\})
$$

is open. Hence, it suffices to show that $\left\{y \in Y: f^{*}(y) \geqq r\right\}$ is closed. Suppose $y_{\alpha} \rightarrow y$ and $f^{*}\left(y_{\alpha}\right) \geqq r$ for some net $y_{\alpha}$ in $Y$. Choose $x_{\alpha} \in X$ so that $\varphi\left(x_{\alpha}\right)=y_{\alpha}$ and $f\left(x_{\alpha}\right)$ $=f^{*}\left(y_{\alpha}\right)$. If $x$ is any limit point of the net $x_{\alpha}$, then $\varphi(x)=y$ and $f(x) \geqq r$, so that $f^{*}(y) \geqq r$.

THEOREM 4.4. If $\varphi$ is an open map of $X$ onto $Y$, then $\pi$ is a weak* open map of $M^{+}(X)$ onto $M^{+}(Y)$.

Proof. Fix $\mu_{0} \in M^{+}(X)$ and let $\nu_{0}=\pi\left(\mu_{0}\right)$. For $\mu \in M^{+}(X), \mu(1)=\|\mu\|$, so that for any $r>\left\|\mu_{0}\right\|$, any weak* neighborhood of $\mu_{0}$ in $M^{+}(X)$ contains one which is contained in $r U(X)$. Hence, for a fixed $r>\left\|\mu_{0}\right\|$, it suffices to show that if $W$ is a weak* compact neighborhood of $\mu_{0}$ in $M^{+}(X)$ and $W \subset K$, where $K=r U(X)$ $\cap M^{+}(X)$, then $\pi(W)$ is a weak* neighborhood of $\nu_{0}$ in $M^{+}(Y)$.

Assume $\pi(W)$ is not a weak* neighborhood of $\nu_{0}$ in $M^{+}(Y)$. Since $\pi$ is weak* continuous, $\pi(W)$ is weak* closed, and since the collection of positive combinations of point masses is weak* dense in $M^{+}(Y)$, there is a net $\nu_{\alpha}$ in $r U(Y) \cap M^{+}(Y)$ which converges weak* to $\nu_{0}$ and is such that, for all $\alpha, \nu_{\alpha} \notin \pi(W)$ and $\nu_{\alpha}$ is a positive combination of point masses in $Y$. Let $A_{\alpha}=\pi^{-1}\left(\nu_{\alpha}\right) \cap K$. Then, by our remarks above, there is a nonempty weak* compact convex set $A$ in $K$ and a subnet $A_{\beta}$ of $A_{\alpha}$ such that $A_{\beta} \rightarrow A$ in the finite topology. Since no $A_{\beta}$ meets the interior of $W$, $\mu_{0} \notin A$. Hence, by the separation theorem, there is an $f \in C(X)$ and $\varepsilon>0$ such that

$$
\mu_{0}(f) \geqq \varepsilon+\mu(f), \quad \text { for all } \mu \in A .
$$

Define $f^{*}$ as in Lemma 4.3. Then $f^{*} \in C(Y)$. Since, for each $\beta, v_{\beta}$ is a positive combination of point masses concentrated at points $y_{\beta, k}$ in $Y$, we may choose corresponding point masses at points $x_{\beta, k}$ in $\varphi^{-1}\left(y_{\beta, k}\right)$ for which $f\left(x_{\beta, k}\right)=f^{*}\left(y_{\beta, k}\right)$, and so construct, for each $\beta$, a $\mu_{\beta} \in M^{+}(X)$ satisfying $\pi\left(\mu_{\beta}\right)=\nu_{\beta}$ and $\mu_{\beta}(f)=\nu_{\beta}\left(f^{*}\right)$. Then $\mu_{\beta}(f) \rightarrow \nu_{0}\left(f^{*}\right)$, and, if $\mu$ is any limit point of the net $\mu_{\beta}$, then $\mu \in A$, since $\mu_{\beta} \in A_{\beta}$ for each $\beta$. Moreover,

$$
\mu(f)=\nu_{0}\left(f^{*}\right)=\mu_{0}\left(f^{*} \circ \varphi\right) \geqq \mu_{0}(f),
$$

which is a contradiction.

5. Examples. In this section we give some examples which help indicate to what extent generalization of the above theorems is not possible. 
Example 1. Let $X=I \times\{0,1\}$ and $Y=I$, where $I$ is the closed unit interval $[0,1]$. Let $\varphi: X \rightarrow Y$ be the projection map, $\varphi(t, i)=t$. Then $\varphi$ is open but the induced map $\pi$ is not weak* open as a map of $U(X)$ onto $U(Y)$. For, if $\delta_{x}$ denotes the unit point mass at $x$, then $\mu_{0}=\frac{1}{2}\left(\delta_{(1,1)}-\delta_{(1,0)}\right)$ is in $U(X)$ and $\pi\left(\mu_{0}\right)=0$. Also, $\nu_{n}=$ $\frac{1}{2}\left(\delta_{0}-\delta_{1 / n}\right)$ converges weak* to 0 in $U(Y)$. But, if $\pi\left(\mu_{n}\right)=v_{n}$ and $\mu_{n} \in U(X)$, then $\mu_{n}$ must be supported on $\varphi^{-1}(\{0,1 / n\})$ and, therefore, cannot converge to $\mu_{0}$.

EXAMPLE 2. Let $E_{1}$ be $R^{3}$ with the norm $\|(x, y, z)\|^{\prime}=\left(x^{2}+y^{2}\right)^{1 / 2}+|z|$ and let $E_{2}$ be $R^{2}$ with the usual norm $\|(x, y)\|=\left(x^{2}+y^{2}\right)^{1 / 2}$. Denote their closed unit balls by $U_{1}$ and $U_{2}$ respectively. Define $\pi: E_{1} \rightarrow E_{2}$ by $\pi(x, y, z)=(x, y+z)$. Then $\pi$ is a linear transformation which maps $U_{1}$ onto $U_{2}$. If $z \neq 0$ and $\|(x, y, z)\|^{\prime}=\|(x, y+z)\|$ $=1$, then $x=0$ and $|y+z|=1$. Therefore, $\{(x, y, z): z>0\} \cap U_{1}$ is a neighborhood of $(0,0,1)$ in $U_{1}$ whose image under $\pi$ contains no points of norm 1 other than $(0,1)$. Hence, on $U_{1}, \pi$ is not open at $(0,0,1)$.

EXAMPLE 3. Let $m$ be Lebesgue measure on $[0,1]$ and let $\mu=m+\delta_{0}$, where $\delta_{0}$ is the unit point mass at 0 . Define $\pi: L_{1}(\mu) \rightarrow L_{1}(m)$ by $\pi(f)=f+f(0)$. Then $\pi$ is a well-defined linear transformation which maps the closed unit ball $U$ of $L_{1}(\mu)$ onto the closed unit ball $U^{\prime}$ of $L_{1}(m)$. Moreover, $\pi$ is positive and is an isometry on positive functions. However, $\pi$ does not preserve intervals nor is $\pi$ open on $U$. We see this as follows. Let $\chi_{A}$ denote the characteristic function of the set $A$ and $\chi_{0}$ the characteristic function of $\{0\}$. Then $\pi\left(\chi_{0}\right)=1$ and, if $f \in L_{1}(\mu)$ and $0 \leqq f \leqq \chi_{0}$, then $f=c \chi_{0}$ for $0 \leqq c \leqq 1$. Hence, $\pi\left[0, \chi_{0}\right] \neq\left[0, \pi\left(\chi_{0}\right)\right]$. Also, on $U, \pi$ is not open at $\chi_{0}$. For $\chi_{[1 / n, 1]} \rightarrow 1$ in $L_{1}(m)$ and, if it is assumed there exist $f_{n} \in U$ such that $\pi\left(f_{n}\right)=\chi_{[1 / n, 1]}$ and $f_{n} \rightarrow \chi_{0}$ in $L_{1}(\mu)$, then $f_{n}(0) \rightarrow 1$ and, in $L_{1}(m)$,

$$
f_{n}=-f_{n}(0) \chi_{[0,1 / n)}+\left(1-f_{n}(0)\right) \chi_{[1 / n, 1]}
$$

so that we eventually have

$$
\begin{aligned}
\int\left|f_{n}\right| d \mu & =f_{n}(0) / n+\left(1-f_{n}(0)\right)(1-1 / n)+f_{n}(0) \\
& =1+(2 / n)\left(f_{n}(0)-\frac{1}{2}\right),
\end{aligned}
$$

contradicting the assumption that every $f_{n} \in U$.

EXAmple 4. Let $X=I \times\{0,1\}$ and $Y=I$ where $I=[0,1]$. Define a linear transformation $\pi$ from $C(X)$ to $C(Y)$ by $\pi(f)(t)=(1-t) f(t, 0)+t f(t, 1)$. Then $\pi$ is a positive operator which maps the closed unit ball $U$ of $C(X)$ onto the closed unit ball $U^{\prime}$ of $C(Y)$. We show that $\pi$ is not open as a map from $U$ to $U^{\prime}$. Let $f_{0} \in U$ be defined by $f_{0}(t, i)=i$ for $(t, i) \in X$. Then $\pi\left(f_{0}\right)(t)=t$ for all $t \in I$. Set $g_{n}(t)=$ $\min (1, t+1 / n)$. Then $g_{n} \in U^{\prime}$ and $g_{n} \rightarrow \pi\left(f_{0}\right)$ in $C(Y)$. Suppose that $f_{n} \in U$ and $\pi\left(f_{n}\right)=g_{n}$. Then, for $1-1 / n \leqq t \leqq 1, g_{n}(t)=1$, so that $f_{n}(t, 0)=f_{n}(t, 1)=1$ and, hence, $\left\|f_{0}-f_{n}\right\| \geqq 1$. Therefore, on $U, \pi$ is not open at $f_{0}$.

6. Selection theorems. By the selection theorems of Michael [3], [4], each of the above open mapping theorems gives rise to a corresponding theorem about continuous selections. 
Let $K(X)$ denote either $U(X)$ or $A \cdot P(X)$, for some fixed set $A$ of real numbers, with the norm topology (or $A \cdot P(X)$ with the weak* topology, if $X$ is metrizable and $\varphi$ is open), and let $K(Y)$ be its counterpart in $M(Y)$.

CoRollary 6.1. Let $S$ be a paracompact space, $S_{0}$ a closed subset of $S$, and $\theta: S \rightarrow K(Y)$ a continuous map. Let $\theta_{0}=\theta \mid S_{0}$, the restriction of $\theta$ to $S_{0}$. Then, for any continuous map $\theta_{0}^{\prime}: S_{0} \rightarrow K(X)$ satisfying $\pi \theta_{0}^{\prime}=\theta_{0}$, there is a continuous map $\theta^{\prime}: S \rightarrow K(X)$ satisfying $\pi \theta^{\prime}=\theta$ and $\theta^{\prime} \mid S_{0}=\theta_{0}^{\prime}$.

Proof. Since $\pi$ is open on $K(X)$ and $\theta$ is continuous, it follows that the map from $S$ to the nonempty closed convex subsets of $K(X)$ which takes $s \in S$ into $\pi^{-1}(\theta(s))$ $\cap K(X)$ is lower semicontinuous. The map $\theta_{0}^{\prime}$ is a continuous selection for this mapping on $S_{0}$. In the norm case, a continuous selection $\theta^{\prime}$ extending $\theta_{0}^{\prime}$ to all of $S$ is guaranteed by [3, Proposition 1.4] and [4, Theorem 1.2] (see also [5, Theorem 1]). In the case of the weak* topology, in $M^{+}(X) \cup M^{-}(X)$ convergence of a net $\mu_{\alpha}$ at the constant function 1 implies convergence of the norms $\left\|\mu_{\alpha}\right\|$, so that pointwise convergence on a dense subset of $C(X)$ containing 1 is equivalent to weak* convergence. Since $C(X)$ is separable when $X$ is metrizable, it follows that the weak* topology of $M^{+}(X) \cup M^{-}(X)$ is metrizable. Moreover, the sets $\pi^{-1}(\theta(s)) \cap K(X)$ are weak* compact. Therefore, in the weak* case, when $X$ is metrizable and $\varphi$ is open [4, Theorem 1.2], still applies.

\section{REFERENCES}

1. M. M. Day, Normed linear spaces, Ergebnisse der Mathematik und ihrer Grenzgebiete, N.F., Heft 21, Academic Press, New York; Springer-Verlag, Berlin, 1962. MR 26 \#2847.

2. E. Michael, Topologies on spaces of subsets, Trans. Amer. Math. Soc. 71 (1951), 152-182. MR 13, 54.

3. - Continuous selections, I, Ann. of Math. (2) 63 (1956), 361-382. MR 17, 990.

4. - A selection theorem, Proc. Amer. Math. Soc. 17 (1966), 1404-1406. MR 34 \#3551.

5. - Selected selection theorems, Amer. Math. Monthly 63 (1956), 233-238.

Department of Mathematics, Louisiana State University, Baton Rouge, Louisiana 70803 